Article

\title{
Change of Basis Transformation from the Bernstein Polynomials to the Chebyshev Polynomials of the Fourth Kind
}

\author{
Abedallah Rababah ${ }^{1,2, *,+}$ (D) and Esraa Hijazi ${ }^{2, \dagger}$ \\ 1 Department of Mathematical Sciences, United Arab Emirates University, 15551 Al Ain, UAE \\ 2 Department of Mathematics, Jordan University of Science and Technology, 22110 Irbid, Jordan; \\ ehhijazi10@sci.just.edu.jo \\ * Correspondence: rababah@uaeu.ac.ae or rababah@just.edu.jo; Tel.: +971-52-4088183 \\ + These authors contributed equally to this work.
}

Received: 6 December 2018; Accepted: 18 January 2019; Published: 23 January 2019

\begin{abstract}
In this paper, the change of bases transformations between the Bernstein polynomial basis and the Chebyshev polynomial basis of the fourth kind are studied and the matrices of transformation among these bases are constructed. Some examples are given.
\end{abstract}

Keywords: Bernstein polynomials; Chebyshev polynomials of fourth kind; computer aided design; basis transformation

PACS: J0101

\section{Introduction}

This paper discusses and finds the matrices of transformation between Bernstein polynomials and Chebyshev polynomials of the fourth kind. Both bases have some excellent advantages and there is a need to convert polynomial forms between them. The Bernstein polynomials form the basis for the Bézier curves and surfaces that are used in modeling and design and in many other applications. The Bézier curves have many geometric properties like the fact that they are modeled by their control points, lying in the convex hull of their control points, and the control polygon is tangent to the Bézier curve. Calculus and degree raising and reduction of the Bézier curves are essential operations in applications. In some cases, the orthogonality property of the Chebyshev polynomials simplifies calculations and enables us to get the solution in explicit form. Thereafter, there is a need to change bases transformations to get the solution in the Bézier form.

An example of a specific essential operation is the Bernstein polynomials [1-3], which are not orthogonal while possessing interesting geometric properties. On the other hand, the Chebyshev polynomials of fourth kind $[4,5]$ are orthogonal, but do not have geometric properties that are in Bernstein polynomials. When the geometric properties are needed, but the curve is given in Chebyshev polynomials of fourth kind, change of bases transformations should be carried out. Some cases are given in [6-8]. These change of bases transformations can also be studied for the cases between the Chebyshev polynomials of fourth kind and the q-Bernstein polynomials in [9], degenerate Bernstein polynomials in [10], and the multidimensional Bernstein polynomials in [11]. This paper is organized as follows. In Section 2, materials, methods, and some preliminary results are stated and proved. In Section 3, the main result of the matrices of change of bases transformations are derived. In Section 4, the proof of the main theorem together with two examples are presented. 
In this section, materials related to this paper are stated. This includes defining the main concepts, giving their properties, and related notations. The Chebyshev polynomials of fourth kind satisfy the following linear homogeneous differential equation of the second order

$$
\left(1-x^{2}\right) y^{\prime \prime}-(1+2 x) y^{\prime}+n(n+1) y=0 .
$$

The Chebyshev polynomials of the fourth kind, $W_{n}(x)$, are orthogonal polynomials on $[-1,1]$ with respect to the weight function $w(x)=\sqrt{\frac{1-x}{1+x}}$. The following recurrence relation is used to acquire the Chebyshev polynomials of the fourth kind:

$$
W_{n}(u)=2(2 u-1) W_{n-1}(u)-W_{n-2}(u), \quad n=2,3,4, \ldots,
$$

where $W_{0}(u)=1$ and $W_{1}(u)=4 u-1, u \in[0,1]$. The Chebyshev polynomials have many applications in science and engineering; the best uniform approximation is characterized by the Chebyshev polynomials of first kind, the weighted least squares approximations are characterized by the relevant weight of Chebyshev polynomials of first, second, third, and fourth kinds. In Computer Aided Design (CAD), the weighted degree reduction of the Bézier curves is handled by the Chebyshev polynomials.For more details about the Chebyshev polynomials of the fourth kind and their applications, see $[4,5,12]$. Other related applications and properties are found in [13-21].

The following notation

$$
B_{i}^{n}(u)=\left(\begin{array}{c}
n \\
i
\end{array}\right)(1-u)^{n-i} u^{i}, \quad u \in[0,1], \quad i=0, \ldots, n
$$

is used for the Bernstein polynomials of degree $n$ over the interval $[0,1]$, where $\left(\begin{array}{c}n \\ i\end{array}\right)=\frac{n !}{i !(n-i) !}$. The Bernstein polynomials are the basis for the Bézier curves and surfaces. The Bernstein polynomials are non-negative $B_{i}^{n}(u) \geq 0, \forall u \in[0,1]$ and are symmetric in the sense $B_{i}^{n}(u)=B_{n-i}^{n}(1-u), \forall u \in$ $[0,1], \forall i=0,1, \ldots, n$. The definite integrals of the Bernstein polynomials $B_{i}^{n}(u), u \in[0,1], i=$ $0,1, \ldots, n$, are given by

$$
\int_{0}^{1} B_{i}^{n}(u) d u=\frac{1}{n+1}, \quad i=0,1, \ldots, n
$$

For more on Bernstein polynomials, see $[2,5]$. The factorial of an integer $n$ is defined by

$$
n !=\left\{\begin{array}{lc}
n(n-1)(n-2) \cdots(2)(1), & n>0 \\
0, & 0 . w
\end{array}\right.
$$

The half-integer factorial is defined as follows:

$$
\left(n-\frac{1}{2}\right) !=\left(n-\frac{1}{2}\right)\left(n-\frac{3}{2}\right) \cdots\left(\frac{3}{2}\right)\left(\frac{1}{2}\right) .
$$

The double factorial of an integer $n$ is defined by:

$$
n ! != \begin{cases}n(n-2) \cdots(4)(2), & \text { if } n \text { even } \\ n(n-2) \cdots(3)(1), & \text { if } n \text { odd }\end{cases}
$$

\section{Materials and Methods}

The following results from $[6,8]$ are used in the proof of the results in this paper. 
For every $n>0$ and $k=0,1, \ldots, n$, we have

$$
\frac{\left(\begin{array}{c}
2 n-1 \\
2 k-1
\end{array}\right)}{\left(\begin{array}{c}
n-1 \\
k-1
\end{array}\right)}=\frac{(2 n-1) ! !}{(2 k-1) ! !(2 n-2 k-1) ! !} .
$$

The combinatorials with an integer plus and minus one half forms satisfy the following relation:

$$
\left(\begin{array}{c}
n-\frac{1}{2} \\
n-k
\end{array}\right)\left(\begin{array}{c}
n+\frac{1}{2} \\
k
\end{array}\right)=\frac{\left(\begin{array}{r}
2 n-1 \\
n
\end{array}\right)\left(\begin{array}{r}
2 n+1 \\
2 k
\end{array}\right)}{2^{2 n-1}} .
$$

The beta function with integer plus one half as parameters has the following equality:

$$
\beta\left(z+\frac{1}{2}, k+\frac{1}{2}\right)=\frac{\pi}{2^{z+k}} \quad \frac{(2 z-1) ! !(2 k-1) ! !}{(z+k) !} .
$$

The Chebyshev polynomial of the fourth kind $W_{n}(u)$ of degree $n$ is expressed in the Bernstein basis $B_{0}^{n}(u), B_{1}^{n}(u), \ldots, B_{n}^{n}(u)$ as follows:

$$
W_{n}(u)=(2 n+1) ! ! \sum_{k=0}^{n} \frac{(-1)^{n-k}}{(2 k+1) ! !(2 n-2 k-1) ! !} B_{k}^{n}(u) .
$$

In the next lemma the integral of the Bernstein and the Chebyshev polynomials of the fourth kind with respect to the weight function $w(u)=\sqrt{\frac{1-u}{u}}$ are found.

Lemma 1. The integral of the product of the Bernstein polynomial of degree $n$ and the Chebyshev polynomials of the fourth kind of degree $j$ is given by:

$$
\begin{gathered}
I_{k j}=\int_{0}^{1} \sqrt{\frac{1-u}{u}} B_{k}^{n}(u) W_{j}(u) d u \\
=\frac{\left(\begin{array}{c}
n \\
k
\end{array}\right) \pi}{2^{2 n+2 j+1}} \sum_{i=0}^{j} \frac{(-1)^{j-1}\left(\begin{array}{c}
2 j+1 \\
2 i+1
\end{array}\right)\left(\begin{array}{c}
2 k+2 i \\
k+i
\end{array}\right)\left(\begin{array}{c}
2 n+2 j-k-2 i+1 \\
n+j-k-i
\end{array}\right)}{\left(\begin{array}{c}
n+j+1 \\
k+i+1
\end{array}\right)} .
\end{gathered}
$$

Proof of Lemma 1. The proof of this lemma is accomplished using Equations (2)-(4) as follows:

$$
I_{k j}=\int_{0}^{1} B_{k}^{n}(u) W_{j}(u)(u)^{\frac{-1}{2}}(1-u)^{\frac{1}{2}} d u .
$$

Using Equation (4) yields

$$
\begin{aligned}
& I_{k j}=\int_{0}^{1} B_{k}^{n}(u)(1-u)^{\frac{1}{2}}(u)^{\frac{-1}{2}}(2 j+1) ! ! \sum_{i=0}^{j} \frac{(-1)^{j-i}}{(2 i+1) ! !(2 j-2 i-1) ! !} B_{i}^{j}(u) d u \\
& =\int_{0}^{1}\left(\begin{array}{c}
n \\
k
\end{array}\right)(1-u)^{n-k}(u)^{k}(1-u)^{\frac{1}{2}}(u)^{\frac{-1}{2}}(2 j+1) ! ! \times
\end{aligned}
$$




$$
\begin{aligned}
& \sum_{i=0}^{j} \frac{(-1)^{j-i}}{(2 i+1) ! !(2 j-2 i-1) ! !}\left(\begin{array}{c}
j \\
i
\end{array}\right)(1-u)^{j-i}(u)^{i} d u \\
= & \int_{0}^{1}\left(\begin{array}{c}
n \\
k
\end{array}\right)(1-u)^{n-k+\frac{1}{2}}(u)^{k-\frac{1}{2}}(2 j+1) ! ! \times \\
& \sum_{i=0}^{j} \frac{(-1)^{j-i}}{(2 i+1) ! !(2 j-2 i-1) ! !}\left(\begin{array}{c}
j \\
i
\end{array}\right)(1-u)^{j-i}(u)^{i} d u \\
= & \left(\begin{array}{c}
n \\
k
\end{array}\right)(2 j+1) ! ! \sum_{i=0}^{j} \frac{(-1)^{j-i}\left(\begin{array}{c}
j \\
i
\end{array}\right)}{(2 i+1) ! !(2 j-2 i-1) ! !} \int_{0}^{1}(1-u)^{n+j-(k+i)+\frac{1}{2}} u^{k+i-\frac{1}{2}} d u .
\end{aligned}
$$

By Equation (3), we get

$$
I_{k j}=\left(\begin{array}{c}
n \\
k
\end{array}\right)(2 j+1) ! ! \sum_{i=0}^{j} \frac{(-1)^{j-i}\left(\begin{array}{c}
j \\
i
\end{array}\right)}{(2 i+1) ! !(2 j-2 i-1) ! !} \beta\left(n+j-k-i+\frac{1}{2}+1, k+i+\frac{1}{2}\right) .
$$

Using Equation (3) yields

$$
\begin{aligned}
I_{k j} & =\left(\begin{array}{c}
n \\
k
\end{array}\right)(2 j+1) ! ! \sum_{i=0}^{j} \frac{(-1)^{j-i}\left(\begin{array}{c}
j \\
i
\end{array}\right)}{(2 i+1) ! !(2 j-2 i-1) ! !} \times \\
& \frac{\pi(2(n+j-k-i+1)-1) ! !(2(k+i)-1) ! !}{2^{n+j+1}(n+j+1) !} \\
& =\frac{\left(\begin{array}{c}
n \\
k
\end{array}\right) \pi}{2^{n+j+1}} \sum_{i=0}^{j} \frac{(-1)^{j-i}\left(\begin{array}{c}
j \\
i
\end{array}\right)(2 n+2 j-2 k-2 i+1) ! !(2 k+2 i-1) ! !(2 j+1) ! !}{(2 i+1) ! !(2 j-2 i-1) ! !(n+j+1) !} .
\end{aligned}
$$

Using Equation (1) gives

$$
\begin{aligned}
I_{k j}= & \frac{\left(\begin{array}{c}
n \\
k
\end{array}\right) \pi}{2^{n+j+1}} \sum_{i=0}^{j} \frac{(-1)^{j-i}\left(\begin{array}{l}
j \\
i
\end{array}\right)(2 n+2 j-2 k-2 i+1) ! !(2 k+2 i-1)\left(\begin{array}{c}
2 j+1 \\
2 i+1
\end{array}\right)}{(n+j+1) !\left(\begin{array}{c}
j \\
i
\end{array}\right)} \\
= & \frac{\left(\begin{array}{c}
n \\
k
\end{array}\right) \pi}{2^{n+j+1}} \sum_{i=0}^{j} \frac{(-1)^{j-i}(2 n+2 j-2 k-2 i+1) ! !(2 k+2 i-1)\left(\begin{array}{c}
2 j+1 \\
2 i+1
\end{array}\right)}{(n+j+1) !} \\
= & \frac{\left(\begin{array}{c}
n \\
k
\end{array}\right) \pi}{2^{3 n+3 j+1}} \sum_{i=0}^{j} \frac{(-1)^{j-i}\left(\begin{array}{c}
2 j+1 \\
2 i+1
\end{array}\right)\left(\begin{array}{l}
2 n+2 j-2 k-2 i \\
n+j-k-i
\end{array}\right)}{1} \times \\
& \frac{\left(\begin{array}{c}
2 k+2 i \\
k+i
\end{array}\right)(2 n+2 j-2 k-2 i) ! !(2 k+2 i) ! !(2 n+2 j-2 k-2 i+1)}{(n+j+1) !} .
\end{aligned}
$$


From the definition of the double factorial of the even integer we get:

$$
\begin{aligned}
I_{k j}= & \frac{\left(\begin{array}{c}
n \\
k
\end{array}\right) \pi^{3 n+3 j+1}}{2_{i=0}^{j}} \frac{(-1)^{j-i}\left(\begin{array}{c}
2 j+1 \\
2 i+1
\end{array}\right)\left(\begin{array}{c}
2 n+2 j-2 k-2 i \\
n+j-k-i
\end{array}\right)}{(n+j+1)} \times \\
& \frac{\left(\begin{array}{c}
2 k+2 i \\
k+i
\end{array}\right)(2 n+2 j-2 k-2 i+1)(n+j-k-i) !(k+i) !}{(n+j) !} \\
= & \frac{\left(\begin{array}{c}
n \\
k
\end{array}\right) \pi}{2^{2 n+2 j+1}} \sum_{i=0}^{j} \frac{(-1)^{j-i}\left(\begin{array}{c}
2 j+1 \\
2 i+1
\end{array}\right)\left(\begin{array}{c}
2 k+2 i \\
k+i
\end{array}\right)}{\left(\begin{array}{c}
n+j \\
k+i
\end{array}\right)} \times \\
& \frac{\left(\begin{array}{l}
2 n+2 j-2 k-2 i \\
n+j-i-k
\end{array}\right)(2 n+2 j-2 k-2 i+1)}{(n+j+1)} .
\end{aligned}
$$

With some more simple calculations, the result is confirmed.

\section{Results}

The matrices of converting the bases between the Chebyshev polynomial of the fourth kind and the Bernstein polynomials are presented in this section. Given a polynomial $P_{n}(u), u \in[0,1]$ written in the Bernstein basis form and in the Chebyshev polynomials of the fourth kind form as follows:

$$
P_{n}(u)=\sum_{j=0}^{n} c_{j} B_{j}^{n}(u)=B_{n} \mathbf{c}_{n},
$$

and

$$
P_{n}(u)=\sum_{k=0}^{n} t_{k} W_{k}(u)=W_{n} \mathbf{t}_{n}
$$

where

$$
\begin{aligned}
B_{n} & =\left(B_{0}^{n}(u), B_{1}^{n}(u), \ldots, B_{n}^{n}(u)\right), \mathbf{c}_{n}=\left(c_{0}, c_{1}, \ldots, c_{n}\right)^{T}, \\
W_{n} & =\left(W_{0}^{n}(u), W_{1}^{n}(u), \ldots, W_{n}^{n}(u)\right), \mathbf{t}_{n}=\left(t_{0}, t_{1}, \ldots, t_{n}\right)^{T} .
\end{aligned}
$$

The $(n+1) \times(n+1)$ matrices of transformation $M$ and its inverse $M^{-1}$ are to be found. They fulfill the following relations:

$$
c_{j}=\sum_{k=0}^{n} M_{j k} t_{k} \text { and } t_{j}=\sum_{k=0}^{n} M_{j k}^{-1} c_{k} .
$$

In matrix form, we have

$$
\mathbf{c}_{n}=M \mathbf{t}_{n} \text { and } \mathbf{t}_{n}=M^{-1} \mathbf{c}_{n} .
$$

The Chebyshev polynomial of the fourth kind $W_{k}(u)$ is presented using the Bernstein polynomials in the following form:

$$
W_{k}(u)=\sum_{j=0}^{n} N_{k j} B_{j}^{n}(u),
$$


where $N$ is the matrix of change of bases transformation of dimension $(n+1) \times(n+1)$. Multiply with $t_{k}$ and take the summation over $k$ to get

$$
\begin{aligned}
\sum_{k=0}^{n} t_{k} W_{k}(u) & =\sum_{k=0}^{n} t_{k} \sum_{j=0}^{n} N_{k j} B_{j}^{n}(u) \\
& =\sum_{j=0}^{n} \sum_{k=0}^{n} t_{k} N_{k j} B_{j}^{n}(u) .
\end{aligned}
$$

Compare it with Equation (5) to get

$$
c_{j}=\sum_{k=0}^{n} t_{k} N_{k j}
$$

Using the following relations $\mathbf{c}_{n}=M \mathbf{t}_{n}$ and $\mathbf{t}_{n}=M^{-1} \mathbf{c}_{n}$ yields

$$
c_{j}=\sum_{k=0}^{n} M_{j k} t_{k}, j=0,1, \ldots, n,
$$

and

$$
t_{k}=\sum_{j=0}^{n} M_{j k}^{-1} c_{j}, k=0,1, \ldots, n .
$$

By comparing with Equation (8), we get $M_{j k}=N_{k j}$; therefore, $M=N^{T}$. The following examples should motivate finding general formulas for these change of basis transformations.

Example 1. For $n=1$ : we have $P_{1}(u)=1 B_{0}+1 B_{1}=1=1 W_{0}+0 W_{1}$. Thus,

$$
C=\left[\begin{array}{l}
1 \\
1
\end{array}\right], T=\left[\begin{array}{l}
1 \\
0
\end{array}\right] .
$$

Since $B_{0}^{1}(u)=1-u, \quad B_{1}^{1}(u)=u$, and $W_{0}(u)=1, \quad W_{1}(u)=4 u-1$, thus

$$
M_{00}^{-1}=\frac{1}{4}, M_{01}^{-1}=\frac{3}{4}, M_{10}^{-1}=\frac{-1}{4}, M_{11}^{-1}=\frac{1}{4} .
$$

Hence

$$
M^{-1} C=\left[\begin{array}{rr}
\frac{1}{4} & \frac{3}{4} \\
\frac{-1}{4} & \frac{1}{4}
\end{array}\right]\left[\begin{array}{l}
1 \\
1
\end{array}\right]=\left[\begin{array}{l}
1 \\
0
\end{array}\right]=T \text {. }
$$

Example 2. For $n=2$, we have $P_{2}(u)=1 B_{0}+1 B_{1}+1 B_{2}=1=1 W_{0}+0 W_{1}+0 W_{2}$. Thus

$$
C=\left[\begin{array}{l}
1 \\
1 \\
1
\end{array}\right], T=\left[\begin{array}{l}
1 \\
0 \\
0
\end{array}\right] .
$$

Since $B_{0}^{2}(u)=u^{2}-2 u+1, \quad B_{1}^{2}(u)=2 u-2 u^{2}, \quad B_{2}^{2}(u)=u^{2}, W_{0}(u)=1, \quad W_{1}(u)=4 u-1$, $W_{2}(u)=16 u^{2}-12 u+1$, thus

$$
\begin{gathered}
M_{00}^{-1}=\frac{1}{8}, M_{01}^{-1}=\frac{1}{4}, M_{02}^{-1}=\frac{5}{8}, M_{10}^{-1}=\frac{-12}{64}, M_{11}^{-1}=\frac{-8}{64}, \\
M_{12}^{-1}=\frac{20}{64}, M_{20}^{-1}=\frac{2}{8}, M_{21}^{-1}=\frac{-1}{8}, M_{22}^{-1}=\frac{-1}{8} .
\end{gathered}
$$


Thus

$$
M^{-1} C=\left[\begin{array}{rrr}
\frac{1}{8} & \frac{1}{4} & \frac{5}{8} \\
\frac{-12}{64} & \frac{-8}{64} & \frac{20}{64} \\
\frac{2}{8} & \frac{-1}{8} & \frac{-1}{8}
\end{array}\right]\left[\begin{array}{l}
1 \\
1 \\
1
\end{array}\right]=\left[\begin{array}{l}
1 \\
0 \\
0
\end{array}\right]=T \text {. }
$$

Theorem 1. The elements of the Matrix $M^{-1}$ that satisfies $B_{n}=W_{n} M^{-1}$ which transforms from the Bernstein polynomial basis into the Chebyshev polynomial basis of the fourth kind for $0 \leq j, k \leq n$ are given by:

$$
M_{j k}^{-1}=\frac{\left(\begin{array}{c}
n \\
k
\end{array}\right)}{4^{n+j}} \sum_{i=0}^{j} \frac{(-1)^{j-i}\left(\begin{array}{c}
2 j+1 \\
2 i+1
\end{array}\right)\left(\begin{array}{c}
2 k+2 i \\
k+i
\end{array}\right)\left(\begin{array}{c}
2 n+2 j-2 k-2 i+1 \\
n+j-k-i
\end{array}\right)}{\left(\begin{array}{c}
n+j+1 \\
k+i+1
\end{array}\right)} .
$$

\section{Discussion}

In this section, the proof of the main theorem is given.

Proof of Theorem 1. In order to get the elements $M_{j k}^{-1}$, we want to find the elements $N_{k i}^{-1}$ first then we get the elements $M_{j k}^{-1}$ by transposing the elements $N_{k j}^{-1}$. We know that

$$
B_{k}^{n}(u)=\sum_{i=0}^{n} N_{k i}^{-1} W_{i}(u)
$$

Multiply the previous equation by $W_{j}(u)\left(\frac{1-u}{u}\right)^{\frac{1}{2}}$ and then integrate from 0 to 1 to get

$$
\int_{0}^{1} B_{k}^{n}(u) W_{j}(u)\left(\frac{1-u}{u}\right)^{\frac{1}{2}} d u=\sum_{i=0}^{n} \int_{0}^{1}\left(\frac{1-u}{u}\right)^{\frac{1}{2}} N_{k i}^{-1} W_{i}(u) W_{j}(u) d u .
$$

By the orthogonality property of the Chebyshev polynomials of the fourth kind, we get

$$
\int_{0}^{1} B_{k}^{n}(u) W_{j}(u)\left(\frac{1-u}{u}\right)^{\frac{1}{2}} d u=N_{k j}^{-1} \int_{0}^{1}\left(\frac{1-u}{u}\right)^{\frac{1}{2}} W_{i}(u) W_{j}(u) d u .
$$

Thus,

$$
\int_{0}^{1} B_{k}^{n}(u) W_{j}(u)\left(\frac{1-u}{u}\right)^{\frac{1}{2}} d u=N_{k j}^{-1} \frac{\pi}{2} .
$$

Therefore,

$$
N_{k j}^{-1}=\frac{2}{\pi} \int_{0}^{1} B_{k}^{n}(u) W_{j}(u)\left(\frac{1-u}{u}\right)^{\frac{1}{2}} d u .
$$

After applying the Lemma we have

$$
N_{k j}^{-1}=\frac{\left(\begin{array}{c}
n \\
k
\end{array}\right)}{2^{n+j}} \sum_{i=0}^{n} \frac{(-1)^{j-i}\left(\begin{array}{c}
j \\
i
\end{array}\right)(2 n+2 j-2 k-2 i+1) ! !(2 k+2 i-1) ! !(2 j-1) ! !}{(2 i-1) ! !(2 j-2 i+1) ! !(n+j+1) !}
$$


and

$$
N_{k j}^{-1}=\frac{2}{\pi} \frac{\left(\begin{array}{c}
n \\
k
\end{array}\right) \pi}{2 \times 4^{n+j}} \sum_{i=0}^{n} \frac{(-1)^{j-i}\left(\begin{array}{c}
2 j+1 \\
2 i+1
\end{array}\right)\left(\begin{array}{c}
2 k+2 i \\
k+i
\end{array}\right)\left(\begin{array}{c}
2 n+2 j-2 k-2 i+1 \\
n+j-k-i
\end{array}\right)}{\left(\begin{array}{c}
n+j+1 \\
k+i+1
\end{array}\right)} .
$$

By transposing $N_{k j}^{-1}$ we get $M_{j k}^{-1}$.

Examples 1 and 2 can be verified using Theorem 1 .

Author Contributions: Conceptualization, A.R.; methodology, A.R.; software, E.H.; validation, E.H.; formal analysis, E.H.; investigation, E.H.; resources, A.R.; writing-original draft preparation, A.R.; writing-review and editing, A.R.; supervision, A.R.; project administration, A.R.

Funding: This research received no external funding.

Acknowledgments: The authors acknowledge the invaluable comments of the reviewers.

Conflicts of Interest: The authors declare no conflict of interest.

\section{References}

1. Farin, G. Curves and Surface for Computer Aided Geometric Design. In Curves and Surface for Computer Aided Geometric Design; Academic Press: Boston, MA, USA, 1993; pp. 32-58, ISBN 9781483296999.

2. Farouki, R.; Rajan, V. Algorithms for polynomials in Bernstein form. Comput. Aided Geom. Des. 1988, 5, 1-26. [CrossRef]

3. Höllig, K.; Hörner, J. Approximation and Modelling with B-Splines. In Approximation and Modelling with B-Splines; SIAM: Philadelphia, PA, USA, 2013; Volume 132, pp. 32-58, ISBN 978-1-611972-94-8.

4. Mason, J.; Handscomb, D. Chebyshev Polynomials. In Chebyshev Polynomials; ACRC Press Company: Miami, FL, USA, 2003; pp. 5-9, 156-158, ISBN 0849303559.

5. Szegö, G. Orthogonal Polynomials. In Orthogonal Polynomials; American Mathematical Society Colloquium Publications: Providence, RI, USA, 1975; ISBN 0821810235.

6. Rababah, A. Transformation of Chebyshev Bernstein polynomial basis. Comput. Meth. Appl. Math. 2003, 3, 608-622. [CrossRef]

7. Rababah, A. Jacobi-Bernstein Basis Transformation. Comput. Meth. Appl. Math. 2004, 4, 206-214. [CrossRef]

8. Rababah, A.; Al-Shbool, A. The Transformation Matrix of Chebyshev IV Bernstein Polynomial Bases. J. Math. Anal. 2016, 7, 123-129.

9. Kim, T. A note on q-Bernstein polynomials. Russ. J. Math. Phys. 2011, 18, 73-82. [CrossRef]

10. Kim, T.; Kim, D. Degenerate Bernstein polynomials. arXiv 2018, arXiv:1806.06379v1.

11. Simsek, Y. Generating functions for unification of the multidimensional Bernstein polynomials and their applications. Math. Methods Appl. Sci. 2018, 41, 7170-7181. [CrossRef]

12. Rababah, A.; Lee, B.G.; Yoo, J. A simple matrix form for degree reduction of Bézier curves using Chebyshev-Bernstein basis transformations. Appl. Math. Comput. 2006, 181, 310-318. [CrossRef]

13. Jang, L.C.; Kim, D.S.; Jang, G.-W.; Kwon, J. Some identities for q-Bernoulli numbers and polynomials arising from q-Bernstein polynomials. Adv. Stud. Contemp. Math. 2018, 28, 659-667.

14. Jang, L.C.; Kim, T.; Kim, D.S.; Dolgy, D.V. On p-Adic Fermionic Integrals of q-Bernstein Polynomials Associated with q-Euler Numbers and Polynomials. Symmetry 2018, 10, 311. [CrossRef]

15. Kim, D.S.; Kim, T.; Ryoo, C.S.; Yao, Y. On p-adic Integral Representation of q-Bernoulli Numbers Arising from two Variable q-Bernstein Polynomials. Symmetry 2018, 10, 451. [CrossRef]

16. Bayad, A.; Kim, T. Identities involving values of Bernstein, q-Bernoulli, and q-Euler polynomials. Russ. J. Math. Phys. 2011, 18, 133-143. [CrossRef]

17. Park, J.-W.; Pak, H.K.; Rim, S.-H.; Kim, T.; Lee, S.-H. A note on the q-Bernoulli numbers and q-Bernstein polynomials. J. Comput. Anal. Appl. 2013, 15, 722-729.

18. Kim, T.; Lee, B.; Lee, S.-H.; Rim, S.-H. A note on q-Bernstein polynomials associated with p-adic integral on Z. J. Comput. Anal. Appl. 2013, 15, 584-592. 
19. Kim, T. A study on the q-Euler numbers and the fermionic q-integral of the product of several type q-Bernstein polynomials on $Z_{p}$. Adv. Stud. Contemp. Math. 2013, 23, 5-11.

20. Kim, T.; Lee, B.; Choi, J.; Kim, Y.H.; Rim, S.H. On the q-Euler numbers and weighted q-Bernstein polynomials. Adv. Stud. Contemp. Math. 2011, 21, 13-18.

21. Ryoo, C.S.; Dolgy, D.V.; Kim, T.; Lee, B. A note on q-Bernstein and q-Laguerre polynomials. Proc. Jangjeon Math. Soc. 2011, 14, 291-298.

(C) 2019 by the authors. Licensee MDPI, Basel, Switzerland. This article is an open access article distributed under the terms and conditions of the Creative Commons Attribution (CC BY) license (http://creativecommons.org/licenses/by/4.0/). 